REVIEW ARTICLE

\title{
Immunization in Rajasthan: Progress, Opportunities, and Challenges
}

\author{
Latika N Sinha ${ }^{1}$, Devinder Sondhi ${ }^{2}$, Kriti Patel ${ }^{3}$
}

\begin{abstract}
Background: In India many newer initiatives are being taken to control vaccine preventable diseases. Introducing new vaccines will help prevent some 1.3 million children deaths attributed to pneumococcal, meningococcal and rotavirus disease. Meticulous monitoring of temperature with newer technologies like electronic Vaccine Intelligence Network (eVIN), enabling real time information on cold chain temperatures, vaccine stocks and flows, use of autodisabled syringes are initiatives for safer service delivery.

Materials and methods: Available literature was reviewed to chronologically describe the newer vaccines introduced in Rajasthan in the last decade. With each vaccine the challenges in coverage were reviewed. Simultaneous availability in the private sector and costs involved have also been stated.

Results: In Rajasthan injectable polio, pentavalent, rotavirus pneumococcal vaccine, measles-rubella are the newer vaccines introduces in the last decade. Positive policy support, sustained funding mechanism, strong collaboration along with a strong and responsive health system have helped to attain coverage of these vaccines among the underserved.

Conclusion: All newer vaccines introduced will have an impact on reducing the infant mortality rate (IMR) and under five mortality rate, if the implementation of routine immunization and acceptance of vaccines is achieved by all concerned stakeholders. Continued support of pediatricians is important to sustain acceptance of vaccines, thus improving routine immunization rates. Much will depend on the continued commitment of the state government and the national and international agencies to sustain and upscale the efforts to meet the child survival goals and to meet the sustainable development goals.

Keywords: Newer vaccines, Rajasthan, Vaccine preventable disease.

Journal of Mahatma Gandhi University of Medical Sciences \& Technology (2018): 10.5005/jp-journals-10057-0071
\end{abstract}

\section{BACKGROUND}

Vaccination is a proven and one of the most cost-effective public health interventions largely responsible for reduction of under-5 mortality rates. ${ }^{1}$ Annually, estimated $2-3$ million deaths and 1 million disabilities are averted by vaccination against diphtheria, pertussis, tetanus, and measles, while another 600,000 future deaths are being prevented by hepatitis $B$ vaccination. Improving services to deliver traditional vaccines will reduce the number of deaths due to vaccine-preventable diseases. In addition, introducing new vaccines will help prevent some 1.3 million children deaths attributed to pneumococcal, meningococcal, and rotavirus diseases. ${ }^{2}$ Palpable success of immunization in eradicating small pox and polio scourge and elimination of maternal and neonatal tetanus (MNTE) resulted in recognition of immense potential of immunization as a public health intervention.

All countries in the world have an immunization program to deliver selected vaccines to the targeted beneficiaries, specially focusing on pregnant women, infants, and children, who are at a high risk of diseases preventable by vaccines. There are at least 27 causative agents against which vaccines are available, and many more agents are targeted for development of vaccines. The number of antigens in the immunization programs has increased in the last 5 years aping the success seen in other countries or in pilot campaigns in India itself.

In India, routine immunization activity gained momentum in late 1970s with launching of the Expanded Programme on Immunization (EPI). The initial list of diptheria, pertussis, tetanus (DPT), oral polio vaccine (OPV), bacillus calmette guerin (BCG), and typhoid-paratyphoid vaccine was later modified to exclude typhoid-paratyphoid vaccine and include tetanus for pregnant
${ }^{1}$ Department of Community Medicine, Government Medical College, Pali, Rajasthan, India

${ }^{2}$ Immunization Division, Directorate of Medical and Health Services, Government of Rajasthan, India

${ }^{3}$ WHO-SEARO Jodhpur Unit, Jodhpur, Rajasthan, India

Corresponding Author: Latika N Sinha, Department of Community Medicine, Government Medical College, Pali, Rajasthan, India, Phone: +91 7728821772, e-mail: drlatika@gmail.com

How to cite this article: Sinha LN, Sondhi D, Patel K, et al. Immunization in Rajasthan: Progress, Opportunities, and Challenges. J Mahatma Gandhi Univ Med Sci Tech 2018;3(2):66-69.

Source of support: Nil

Conflict of interest: None

mothers in 1983. The program was modified in 1985 as the Universal Immunization Programme (UIP) to cover the entire country in a phased manner. Measles vaccination was included in 1985, and vitamin A supplementation was added in 1990. India has one of the largest UIPs in the world in terms of number of beneficiaries (27 million infants and 30 million pregnant women), quantities of vaccines used, geographical spread, and manpower involved. ${ }^{3}$

Besides newer vaccines, there have been commendable developments in newer technologies that may greatly influence the quality of immunization services. A vaccine vial monitor (VVM) is one such simple and effective tool, which is used by health workers to detect cold chain failure, especially during outreach sessions in remote areas. Meticulous monitoring of temperature with newer technologies like the electronic Vaccine Intelligence Network (eVIN), enabling real-time information on cold chain temperatures and

(c) The Author(s). 2018Open Access This article is distributed under the terms of the Creative Commons Attribution 4.0 International License (https://creativecommons. org/licenses/by-nc/4.0/), which permits unrestricted use, distribution, and non-commercial reproduction in any medium, provided you give appropriate credit to the original author(s) and the source, provide a link to the Creative Commons license, and indicate if changes were made. The Creative Commons Public Domain Dedication waiver (http://creativecommons.org/publicdomain/zero/1.0/) applies to the data made available in this article, unless otherwise stated. 
vaccine stocks and flows, has immensely benefit the program. It has proved a boon for safeguarding the cold chain system at different storage points especially for freeze-sensitive vaccines like HepB, DPT, DT, and TT in addition to tracking temperature maintenance till the point of delivery. 4

Another novel advancement is the introduction of auto disabled syringes (ADS). Introduction of ADS has resolved the problems of sterilization, particularly in resource-poor settings and difficult areas, and ensures injection safety to a great extent. ${ }^{4}$

In spite of all these newer initiatives to control vaccinepreventable diseases, the challenge remains to transmit the benefits of scientific developments to all. The greatest challenge is to make services available particularly to the vulnerable section of population.

To overcome these challenges, WHO and UNICEF recommended the main strategic areas in the Global Immunization Vision and Strategy, 2006-2015. ${ }^{5}$

- Immunize "hard-to-reach" infants and other age groups through a focus on the district level.

- Increase the availability and affordability of new life-saving vaccines.

- Deliver key health interventions, including insecticide-treated nets against malaria, vitamin A supplements, and deworming medicine during immunization contacts, especially for the "hard to reach."

- Strengthen cross-border collaboration and coordination to ensure a reliable supply of vaccines, sustainable financing of vaccination, and epidemic preparedness.

In Rajasthan, about 1.8 million pregnant women (PW) and 1.6 million children are targeted every year using more than 21 million syringes. ${ }^{6}$ The latest vaccines introduced in Rajasthan state include injectable polio vaccine (IPV), rotavirus vaccine (RVV), pneumococcal conjugate vaccine (PCV), and the recently launched measles-rubella (MR) vaccine.

Rajasthan is one of the few states in the country that has shown an increasing trend in evaluated immunization coverage. As per coverage evaluation survey (CES) 2009 data, percentage of fully immunized children was $53.8 \%$ in the state. As per annual health survey (AHS) 2012-13 data, the fully immunization coverage evaluated $74.2 \%{ }^{7}$ As per the current survey conducted by the UNICEF, FI coverage is $77.8 \% .^{8}$

Rajasthan has geared up the priority action points as per the Multi-Year Strategic Plan (2013-17) in the following areas: ensuring regularity of sessions, adequate staffing, monitoring, safe injection, improved cold chain and vaccine logistics management, and social mobilization. ${ }^{9}$ Other identified areas of concern are diverse geographical area-desert and tribal belt with poor coverage, very small hamlets, hard-to-reach villages, lack of human resources, false beliefs, lack of monitoring, high dropouts, cold chain management, surveillance of vaccine preventable disease, maintenance of equipment, etc.

On the one hand, there are so many gaps and bottlenecks in the program management; on the other hand, there are numerous newer vaccines in the pipeline opening up newer opportunities to reduce child deaths. This is a peculiar juncture considering National Technical Advisory Group on Immunization (NTAGI) recommendations, which explored the scope of newer vaccine inclusion. ${ }^{10,11}$ Developing a strong delivery system is of utmost importance to ensure availability and accessibility of quality immunization services.

\section{Newer Vaccines in Rajasthan} IPV

Participating in the Global Polio Endgame Strategy (2013-2018), by introducing a highly effective IPV, in addition to OPV in routine immunization, it was introduced on November 30,2015, and globally synchronized switch activity from trivalent oral polio vaccine (tOPV) to bivalent oral polio vaccine (bOPV) was achieved on April 25, 2016, with Global Alliance for Vaccine Initiative (GAVI) and NTAGI that recommended IPV introduction as an additional dose along with third dose of DPT in the entire country in the first quarter of $2016 .{ }^{11}$ This was followed by introduction of the fractional intradermal dose IPV (f-IPV) in two doses at 6 weeks and 14 weeks replacing the single-dose intramuscular schedule. As IPV is an inactivated vaccine, it carries no risk of vaccine-associated paralytic polio (VAPP) and vaccine-derived polio virus (VDPP). Injectable polio vaccine provides excellent humoral immunity and also boosts intestinal mucosal immunity in OPV-primed children. Recent studies in India and Bangladesh have shown that two f-IPV intradermal doses provide similar levels of protection as compared with one full intramuscular IPV dose. ${ }^{12}$ The primary role of IPV will be to maintain immunity against type II poliovirus while removing all polio virus serotypes globally.

\section{RVV}

Diarrheal diseases are second leading cause of death in under-5 children. Rotavirus is the most common cause of severe diarrhea in children. Approximately $50 \%$ of rotavirus-associated deaths occur in the first year of life, and about $75 \%$ occur in the first two years of life. It also accounts for $40 \%$ of hospitalizations due to diarrhea in children. ${ }^{13}$ No specific therapy is currently available.

In India, around 1.7 million under-5 children die every year, and $13 \%$ of these deaths are due to diarrhea. Moreover, in India, rotavirus is responsible for nearly (annually, in under-5 children) around 8.70 lakh inpatient admissions and 32.7 lakh outpatient visits along with 78,000 deaths.

The use of RVVs is a part of an integrated approach to control diarrheal diseases with both prevention (promotion of early and exclusive breastfeeding, handwashing, improved water supply, and sanitation) and treatment strategies.

As per the National Family Health Survey (NFHS-4) data, in Rajasthan 7.4\% of children under age 5 years were found with diarrhea within 2 weeks preceding the survey. The survey shows, among such children, that $73.9 \%$ were suffering from diarrhea in past 2 weeks of survey who were taken to hospital for treatment.

The rotavirus vaccine was introduced in the state in March 2017. The vaccine is given orally to all children at 6,10 , and 14 weeks of age as five drops dose till 1 year of age. Immunization is the only specific strategy to prevent rotavirus diarrhea among children. ${ }^{2}$ In Rajasthan, 17.25 lakh children benefited with the introduction of vaccine. ${ }^{6}$

The vaccine is available in the private sector at a cost of Rs. 3,000 for a full course but is now provided free of cost to children in the state.

\section{PCV Vaccine}

Pneumonia is number one killer among leading infectious diseases in the under-5 age group in India. Around 10 lakh deaths take place every year in the world, of which $16 \%$ in under- 5 were due to pneumonia. ${ }^{14}$ India shares $20 \%$ of global deaths due to pneumonia, 
according to the World Health Organization and Maternal Child Epidemiology Estimation, 2017.

Two primary doses of PCV are administered to children at 6 weeks and 14 weeks and a booster dose at the ninth month. PCV reduces incidence of pneumonia, meningitis, and other serious bacterial infections caused by pneumococcal bacteria. It is a very expensive vaccine, available in private sector and three doses of it cost around Rs. 10,000-12,000, which only a few can afford. By including it in the routine immunization program, the underprivileged and poor people will get it administered to their children free of cost.

Vaccine industry is one of the fastest growing industry and between 2000 and 2008, the global vaccine market almost tripled to $\$ 17$ billion. A total of 35 countries introduced PCV in the routine program. In Rajasthan, the under- 5 mortality rate is 45 as per the Sample Registration System (SRS), 2016; in 2015, the rate was 50. The PCV vaccine was first introduced in nine districts Banswara, Barmer, Dungarpur, Jalore, Pali, Pratapgarh, Rajsamand, Sirohi and Udaipur which have high under-5 mortality in Rajasthan. It is planned to be introduced in remaining districts of Rajasthan till 2019 end.

\section{MR Vaccine}

Measles is a highly infectious and potentially fatal disease with complications like pneumonia, diarrhea, blindness, and encephalitis, which may lead to death among children. Globally, more than 1 lakh children die annually due to measles and of these 49,000 are from India. ${ }^{15}$

Rubella infection, although mild, can lead to fetal death, spontaneous abortion, and still birth during early pregnancy. It also causes a set of serious birth defects known as congenital rubella syndrome (CRS) consisting of ear, eye, heart, and liver problems and mental retardation resulting in huge burden to family and society. ${ }^{16-18}$

India is committed to the goal of measles elimination and control rubella/CRS by 2020. The Government of India launched a massive public health initiative, the MR vaccination campaign in February 2017 in a phased manner-one of the world's largest ever vaccination campaign to cover 41 crore children in the age group of 9 months to less than 15 years. ${ }^{1}$ More than 30.5 crore children in 14 states of India have already been covered till May 2019.

This additional campaign dose will boost the immunity of child and protect the entire community by eliminating transmission of measles and rubella. The conduct of measles-rubella (MR) vaccination campaigns was an important factor in achieving measles elimination in the Western Hemisphere (2002) and the elimination of indigenous rubella in $2009 .{ }^{19}$ The campaign also marks the introduction of the rubella vaccine in India's childhood immunization program to address CRS that causes birth defects such as irreversible deafness and blindness in nearly 40,000 children every year.

Within a span of 4-6 weeks, vaccination will be conducted in schools during first 2-3 weeks and later on in the community through outreach sessions. Currently, this vaccine is being given to all targeted children, irrespective of prior MR immunization status or disease status. With the target of greater than $95 \%$ coverage, to knock out the susceptible cohort, this vaccine will replace the measles vaccine in the routine immunization schedule. It will not allow virus to circulate freely and infect women of child-bearing age, thus avoiding any inadvertent epidemiological shift/cross infection. Measles-rubella vaccine is the most recent vaccine introduced on July 22, 2019, in Rajasthan with a target to cover 2.26 crore children in 33 districts of Rajasthan. ${ }^{20}$
All newer vaccines introduced will have an impact on reducing the infant mortality rate (IMR) and under- 5 mortality rate, if the implementation of routine immunization and acceptance of vaccines are achieved by all concerned stakeholders. The challenges facing researchers, industry, and policymakers are many, but optimism abounds. Experts believe that immunology is on the cusp of a renaissance.

Pediatricians and immunization providers are among the key opinion leaders at the national and state level and also have a voice in local communities. Therefore, continued support of pediatricians is important to sustain acceptance of vaccines, thus improving routine immunization rates. ${ }^{3}$ Positive policy support, sustained funding mechanism, and strong collaboration along with a strong and responsive health system are necessary to attain the goal of universal coverage.

Much will depend on the continued commitment of the state government and the national and international agencies to sustain and upscale the efforts to meet the child survival goals and the sustainable development goals.

\section{References}

1. Lahariya C. A brief history of vaccines \& vaccination in India. Indian J Med Res 2014;139(4):491-511, http://www.searo.who.int/india/topics/ measles/faqs_measles_rubella_vaccine_english.pdf (last accessed on 20/08/2017).

2. htttp://www.who.int/immunization/newsroom/en/last accessed 25/07/2019.

3. Family Welfare Statistics in India, MoHFW, Govt. of India, 2006.

4. http://www.in.undp.org/content/india/en/last accessed 25/07/ 2019.

5. WHO-UNICEF. WHO Department of Immunization, Vaccines and Biologicals and UNICEF Programme Division, Health Section www. who.int/vaccines-documents/www.unicef.org Global Immunization and Vaccine strategy, 2006-15.

6. www.indiastats.com/healthdata/16/Immunization/128/stats.

7. Annual Health Survey report, Ministry of Home Affairs, Rajasthan Factsheet 2013-14.

8. www.unicef.org/about/execboard/files/2019_AS.../last accessed 26/07/2019.

9. MOHFW GOI Multi Year Strategic Plan Reaching every child 2013-17.

10. Background material for NTAGI Standing Technical Sub-Committee (STSC) Meeting on "Potential Strategies for the Control of Rubella and CRS Burden in India." February 26, 2014; Indian Council of Medical Research, New Delhi.

11. Sutter R, Bahl S, et al. Immunogenicity of a new routine vaccination schedule for global poliomyelitis prevention: an open-label, randomised controlled trial. The Lancet December 12 2015; 386(10011):2413-2421. DOI: 10.1016/S0140-6736(15)00237-8.

12. Sarkar R, Muliyil J, et al. Rotavirus gastroenteritis in India, 2011-2013: Revised estimates of disease burden and potential impact of vaccines. Vaccine 2014;32(1):A5-A9. DOI: 10.1016/j.vaccine.2014.03.004.

13. Farooqui $\mathrm{H}$, Jit M, et al. Burden of Severe Pneumonia, Pneumococcal Pneumonia and Pneumonia Deaths in Indian States - Modelling Based CHERG Estimates. PLoS ONE 10(6):e0129191. DOI: 10.1371/ journal.pone.0129191.

14. Government of India, Ministry of Health and Family Welfare (Immunization Division). ICMR Expert Group Recommendations on MR campaign ICMR, Feb 2017.

15. WHO Position Paper, Mumps Virus Vaccines. Wkly Epidemiol Rec 2019;82:49-60.

16. Dewan P, Gupta P. Burden of congenital rubella syndrome (CRS) in India: A systematic review. Indian Pediatr 2012;49:377-399. DOI: 10.1007/s13312-012-0087-4. 
17. Background material for NTAGI Standing Technical Sub-Committee (STSC) Meeting on "Potential Strategies for the Control of Rubella and CRS Burden in India." February 26, 2014; Indian Council of Medical Research, New Delhi.

18. Vashishtha VM, Yewale VN, et al. IAP perspectives on measles and rubella elimination strategies. Indian Pediatr 2014 Sep 1;51(9): 719-722. DOI: 10.1007/s13312-014-0488-7.
19. Status Report on Progress towards Measles and Rubella Elimination. SAGE Working Group on Measles and Rubella (17 October 2013) Available from: http://www. who.int/immunization/ sage/meetings/2013/november/Status_Report_Measles_ Rubella21Oct2013_FINAL.pdf Accessed on May 19, 2014.

20. timesofindia.indiatimes.com/city/jaipur/measles-rubella vaccine campaign. 